LA-UR- - $92-1327$

$$
\operatorname{louf} \cdot 12(1214)-3
$$

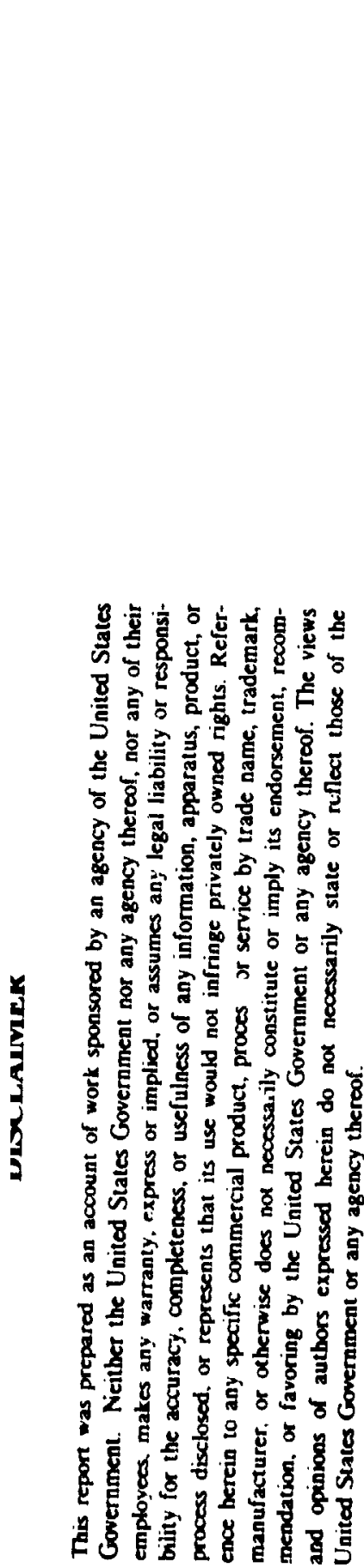

LA-UR--92-1327

DE92 013532

Author(s):

Results from Beam Tests of MEGA's Low-Mass, High-

Rate Cylindrical MWPCs

Submitted to:

S. Stanislaus, V. Armijo, J. K. Black, R. D. Bolton,

S. Carius, M. D. Cooper, C. Espinoza, G. Hart,

G. Hogan, A. Gonzales, R. E. Mischke, L. E. Piilonen, $J$. Sandoval, S. Schilling, J. Sena, G. Suazo,

J. J. Szymanski, D. A. Whitehouse, C. A. Wilkinson,

R. Fisk, D. D. Koetke, R. W. Manweiler, and C. C. Jui

The 6th Wire Chamber Conference

Vienna, Austria, February 17-21, 1992

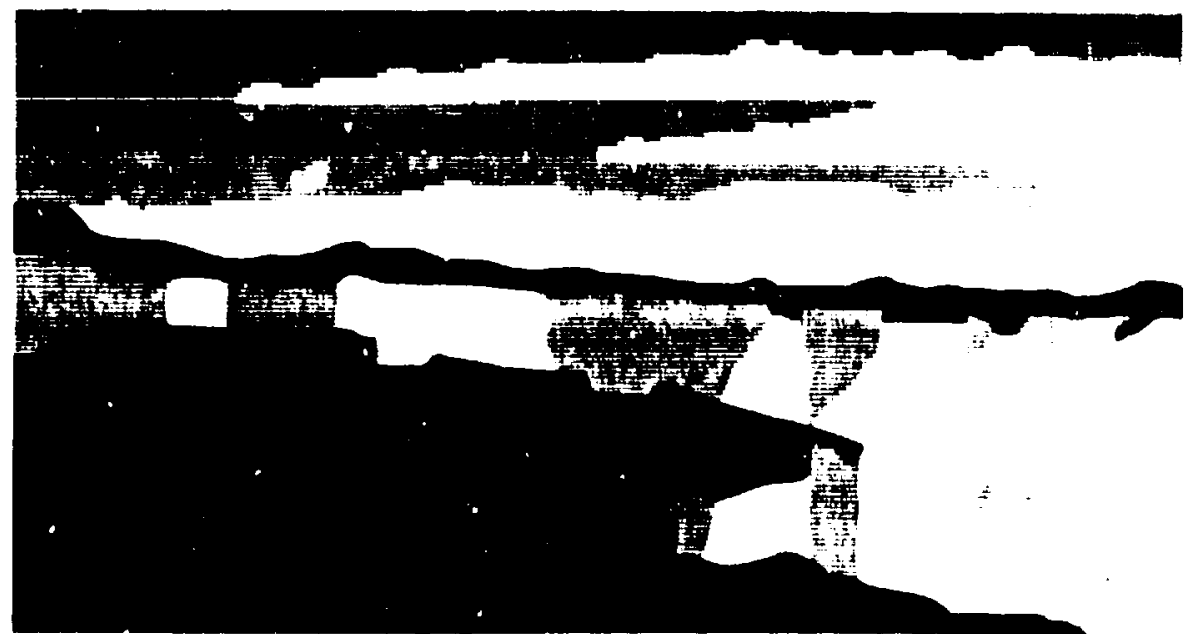

NATIONAL LABORATOAY

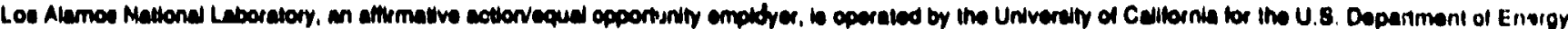

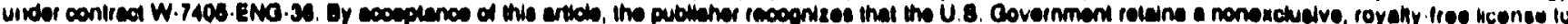

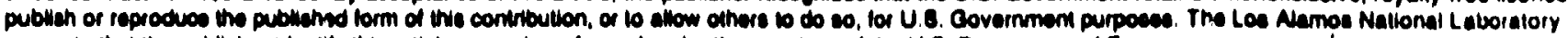

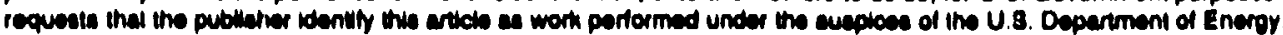




\title{
Results from Beam Tests of MEGA's Low-Mass, High-Rate Cylindrical MWPCs*
}

S. Stanislaus, V. Armijo, J.K. Black, R.D. Bolton, S. Carius, M.D. Cooper, C. Espinoza, G. Hart, G. Hogan, A. Gonzales, R.E. Mischke, L.E. Piilonen, J. Sandoval, S. Schilling, J. Sena, G. Suazo, J.J. Szymanski, D.A. Whitehouse, C.A. Wilkinson(Los Alamos National Laboratory)

R. Fisk, D.D. Koetke, R.W. Manweiler (Valparaiso University) C.C. Jui (Stanford University)

\begin{abstract}
One of the leading experimental projects at LAMPF has been the MEGA experiment. This is an experiment to search for the rare decay $\mu \rightarrow$ ey with a sensitivity of $10^{-13}$. A prime component of this project has been the design and construction of high-rate, lon mass MWPCs for the tracking of positrons from muon decay. With rate capabilities of $2 \times 10^{4} \mathrm{e}^{+} / \mathrm{mm}^{2} / \mathrm{s}$ and a thickness of $3 \times 10^{-4}$ radiation lengths, these chambers are state-of-the-art cylindrical MWPCs. Cylindrical chambers of this size $\left(0.9 \mathrm{~m}^{2}\right)$ and thinness have never been previously constructed. The MEGA project at LAMPF has recently succeeded in building chambers with these necessary performance characteristics as demonstrated by data taken from muon decays, cosmic rays, and sources.
\end{abstract}

- Work supported by the U.S. Department of Energy. 


\section{Introduction}

MEGA is the latest in a series of muon decay experiments at LAMPF. It is designed to search for the rare decay $\mu^{+} \rightarrow \mathrm{e}^{+}+\gamma$ with a sensitivity of a few parts in $10^{13}$. The MEGA detector is a magnetic spectrometer designed to detect in coincidence the back-to-back 52.8 Mel' positron and the 52.8 MeV photon from the decay of the muon stopped in a thin target at the center of the spectrometer. The photon detector consists of three concentric, cylindrical pair spectrometers. The positron detector consists of eight state-of-the-art low-mass, high-rate cylindrical MWPCs for high precision tracking, and two cylindrical banks of 90 scintillators each to obtsin precise timing. The photon detector surrounds the positron detector, and the entire apparatus is contained in a superconducting solenoid of $1.5 \mathrm{~T}$. Details of the design of the experiment can be found in references [1-3]. This paper will discuss test results from the positron arm MWPCs [3]. Details of the chamber construction and elecironics will be the subject of a later paper.

\section{Design Considerations for Positron MWPCs}

There were two dominant considerations in the design of the positron MWPCs. First, we seeded low mass to minimize positron interactions producing $\gamma$ rays. This property also aids in achieving good positron energy resolution $(0.6 \%$ at $52.8 \mathrm{MeV}$ ) by minimizing multiple scattering. Second, we needed stable and efficient operation in an instantaneous $\mu$ stopping rate of $500 \mathrm{MHz}$ for more than $10^{7}$ seconds.

The actlve chamber region consists of two concentric cylindrical cathode foils with the anode wires stretched axially between the two foils. Wires and foils are attached to two end pleces, that are in turn attached to a fixture (the tension shell) that holds all the chambers and maintains wire tension. The cylindrical foil shape is supported by differential gas pressure. The thickness of the chamber (two foils, gas 
and wires) for normal incidence is $3 \times 10^{-4}$ radiation lengths. A geometrical configuration that has been adopted to minimize the occupancy rate is shown in Fig. 1. In this configuration the chambers are named Snow White (SW, the larger chamber) and the seven dwarfs. The maximum flux in a chamber is expected to be 2 $\times 10^{4} \mathrm{e}^{+} / \mathrm{mm}^{2} / \mathrm{s}$.

Some of the chamber parameters are: wire spacing $1 \mathrm{~mm}$, half-gap $1.75 \mathrm{~mm}$, length $126 \mathrm{~cm}$, dwarf radius $5.982 \mathrm{~cm}$, SW radius $11.138 \mathrm{~cm}$, wires $15 \mu \mathrm{m} \mathrm{Au}-W$, cathode foil $200 \mathrm{~nm}$ Cu on $25 \mu \mathrm{m}$ kapton, cathode stripe width $2.7 \mathrm{~mm}$, gas $\mathrm{CF}_{4}(80 \%)$ + isobutane(20\%), and four electro-mechanical supports. The supports each consist of a $0.1 \mathrm{~mm}$ diameter moriofilament over the wires and a $0.66 \mathrm{~mm}$ diameter glass cane under the wires.

\section{The Test Run}

Engineering tests were conducted on the fesitron MWPCs during a 1990 beam run to demonstrate that the chambers would meet the needed requirements. Because the design work and code development relied heavily on the Monte Cario code used to simulate the chamber response, we needed to compare the observed characteristics of the MWPCs with those of the simulation. The important features to be verified include the energy and position resolutions of the chambers as well as the degree of occupang. The chamber tests were conducted with a limired protstype system consisting of a complete barrel of 90 position scintillators, a fully instrumenter dwarf chamber with $1 \mathrm{~mm}$ wire spacing, a $5 W$ chamber with $2 \mathrm{~mm}$ wire spacing, and one layer of scintillators in the photon arm.

\section{Chamber Stability and Timing Teat}

During bench tests using a ${ }^{90} \mathrm{Sr}$ source producing the same current density as the beam, it was observed that at about $100 \mathrm{~V}$ below the operating voltage of $2450 \mathrm{~V}$ 
the dwarf chambers produced a dark current of $\sim 1-100 \mu \mathrm{A}$ that was not self quenching when the source was removed. The purpose of the larger wire spacing used in the SW was to address this problem. The SW chamber did not produce dark currents during bench tests. The prototype chambers reproduced the same behavior when illuminated by positrons from muon decays as when exposed to the ${ }^{90} \mathrm{Sr}$ source. The dwarf chamber was unstable above $2350 \mathrm{~V}$ at rates above $1 \mathrm{MHz}$ whereas the SW chamber ran without trouble at full voltage $(1850 \mathrm{~V})$ even when the beam rate was $240 \mathrm{MHz}$. On the basis of this we concluded that the chamber dark current problem was not related to the gas gain.

The intrinsic drift time distribution had previously been nieasured for a prototype chamber that had short $(30 \mathrm{~cm})$ wires with the same wire spacing and half gap as the dwarf chambers. These tests showed that for a chamber using a mixture of $80 \% \mathrm{CF}_{4}+20 \%$ isobutane the collection time was $12 \mathrm{~ns}$ (base width) [2]. During the 1990 run, timing measurements were made to study the characteristics of anode and cathode pulses. No difference was seen in the transmission properties between wires and stripes; there was no evidence of broadening in SW timing as the result of the wider spacing.

Figure 2 shows a typical cosmic ray time spectrum. It shows the time difference between the trigger scintillators and the first anode signal. In addition to the drift time, this spectrum contains contributions from jitter in the trigger box, track time-of-fight broadening, leading edge slewing from variations in pulseheight, and variation in propagation time due to location of the chamber hit. With these additional components, the resulting distribution of arrival times at the TDC should roughly double in width from that of the collection time. The spectrum shown in Flg. 2 with FWHM of $10 \mathrm{~ns}$ is therefore consistent with these expectations. It is important for the $\mu \rightarrow$ ey search that the distribution of arrival times be made as 
narrow as possible since a broader spectrum would result in increased accidental background in the chambers.

\section{Comparison with Monte Carlo}

We have developed a detailed Monte Carlo of the MWPCs including effects such as multiple scattering, variable eneigy deposition, pulse shape effects, etc. Using the parameters given in sections 2 and 3 the results of the simulation are compared with the 1990 data.

\subsection{Hit Multiplicities}

In addition to the rate of incident particles, the occupation fraction of the chambers depends on the number of wires or stripes activated by each particle. For the anodes, the multiplicity is determined mainly by geometry, i.e. the numbar of cells crossed by a given track. The situation is slightly more complicated for cathodes, where a realistic model of the image charge distribution is needed to make a correct simulation of hit multiplicities. The cathode multiplicity was one aspect of the Monte Carlo code that needed verification. In addition, the code does not include cross-talk between neighboring channels.

Figure 3 shows the histograms of anode and cathode hit multiplicity vs. incident angle of straight tracks (magnetic field off) on the chamber. The angle used here is that between the $x-y$ projection (Fig. 1) of the track and a vector normal to the axis of the chamber; $0^{\circ}$ represents a track incident along the normal. Events with angles up to $80^{\circ}$ are included. Beyond this value, separate clusters in the cliamber cannot be resolved. The anode distributions are in excellent agreement between the experiment and the Monte Carlo, where at very large angles the data exceed the simulation results by about $10 \%$. The discrepancies were somewhat larger for the cathodes, where the data are $\sim 20 \%$ higher over the wide 
range of angles. This might indicate a significant contribution from cross-talk. The level of agreement is adequate for MEGA's needs. Similar data taken with the magnetic field on (curved tracks) show that the magnetic field does rot affect the results.

\subsection{Position Resolution}

Straight track events (muon decays with magnet off) were used to make a comparison between the observed and expected position resolutions of the chambers. A straight track crosses SW at one point and the dwari at two points. The dwarf chamber hits are used to predict the location of the crossings in SW. Histograms of the difference between the projected SW crossings in $\phi$ and $z$ values (in a cylindrical coordinate system centered at SW), and those obtained from the SW chamber hits are shown in Fig. 4. For both $\Delta \phi$ and $\Delta z$, the simulation is in excellent agreement with experiment. Hence the position resolution of the chambers are reproduced accurately by the simulation program.

\subsection{High Rate Tests}

The onset of dark currents in the dwarf chsmber restricted the high rate tests to $S W$ and positron scintillators. Data were taken at various beam intensities up to an instantaneous rate of $240 \mathrm{MHz}$. Occupancy rates were studied by looking at the average number of anode and cathode clusters as a function of the beam rate. While the observed anode rate was slightly above the simulated rate, the experimental cathode rate was significantly below the Mionte Carlo values. We attribute the larger discrepancy in the cathode rates to the absence of pulse-shaping in the cathode readout electronics [3]. The discrepancies in the results are within tolerable levels. We expect the level of agreement to improve with the installation of pulse-shaping on the amplifier-discriminator cards. 


\subsection{Energy Resolution and Track Reconstruction}

During the latter part of the run sixteen hours were devoted to a trial search of $\mu \rightarrow$ eq. Approximately 4000 background positrons were reconstructed from this data. The energy spectrum of these events is shown in Fig. 5 along with a Monte Carlo Michel spectrum. The agreement is very good between the two spectra showing that the simulation reliably predicts the energy resolution of the detector. As another check of the simulation, Fig. 6 shows the fraction of events containing a reconstrucied background track plotted against the instantantous beam rate. The linear fit to the data points shows that as expected, at these rates the observations fit quite well to a linear model. Two Monte Carlo points are also shown in the plot, and are in very good agreement with the data points.

\section{Progress Since The 1990 Run}

At the end of the 1990 summer run the single outstanding technical issue remaining was the rate irduced dark current. Tests revealed that the dark currents were usually drawn at the support posts of the glass garlands. The posts were replaced with glass beads. The wire spacing was also increased from $1 \mathrm{~mm}$ to $1.1 \mathrm{~mm}$, bringing the operating voltage down to $2325 \mathrm{~V}$. The above changes improved the stability of the chambers, but still they coulld not stand the full rate. The addition of $0.5 \%$ of isopropanol to the gas mixture made them stable and capable of handling the full rate of the experiment. But when the alcohol flow was stopped, the performance of the chambers was worse than before, and showed signs of degradation from the isopropanol. Subsequently, the wire spacing was increased to $1.3 \mathrm{~mm}$. With the use of $0.2 \%$ water as the quenching agent instead of iscpropanol, the chambers perform very reliably and show no signs of degradation. With the increased wire spacing the operating voltage is reduced to $2150 \mathrm{~V}$. Monte 
Carlo studies have shown that the larger wire spacing has negligible impact on tracking resolution, but will increase the occupancy fraction of the chambers making it necessary to lower the beam rate by about $10 \%$.

\section{Summary}

After several years of detector development, prototypes of MEGA's high-rate, low-mass positron MWPCs were successfully tested with beam in 1990. Apart from the dark current problem (which was solved after the run), the behavior of the chambers were in good agreement with predictions of the simulation. In particular, the observed hit multiplicity and background cluster rates agree with simulated values at the $20 \%$ level. Within the limitations of the test, the observed clamber resolutions are identical to the Monte Carlo values.

The construction of all eight MWPCs is complete. The MEGA collaboration expects to search $\mu \rightarrow$ ey to $6 \times 10^{-12}$ level in the summer of 1992 and to reach $6 \times 10^{-13}$ by the summer of 1994 .

\section{References}

[1] M.D. Cooper, in: Intersection between Particle and Nuclear Physics, ed. G.E. Bunce (AIP, New York, 1988) p. 845; D.D. Koetke, in: Intersection between Particle and Nuclear Physics, ed.W.T.H. Van Oers (AIP, New York, 1991) p.397; LAMPF experiment 969, M.D. Cooper spokesman.

[2] V. Armijo et. al , Nucl. Instr. and Meth. A303 (1991) 298.

[3] C.C. Jui; Ph.D. thesis, Stanford University , 1992.

\section{Figure Captions}

1. Cross section of positron arm perpendicular to the spectrometer (B-field) axis.

2. Time spectrum for MWPC signals 
3. Comparison of hit multiplicity vs. track incident angle for data and simulated events. The average multiplicities are shown in the profile on the right of each plot.

4. Comparison of experimental and Monte Carlo distributions for the difference between projected and actual $\phi$ (top) and $z$ (bottom) coordinates of hits in SW from straight tracks.

5. Comparison of energy spectra of background Michel events from ey data set with Monte Carlo events.

6. Plot of number of reconstructed background tracks per 1000 triggers vs. instantaneous beam rate. 


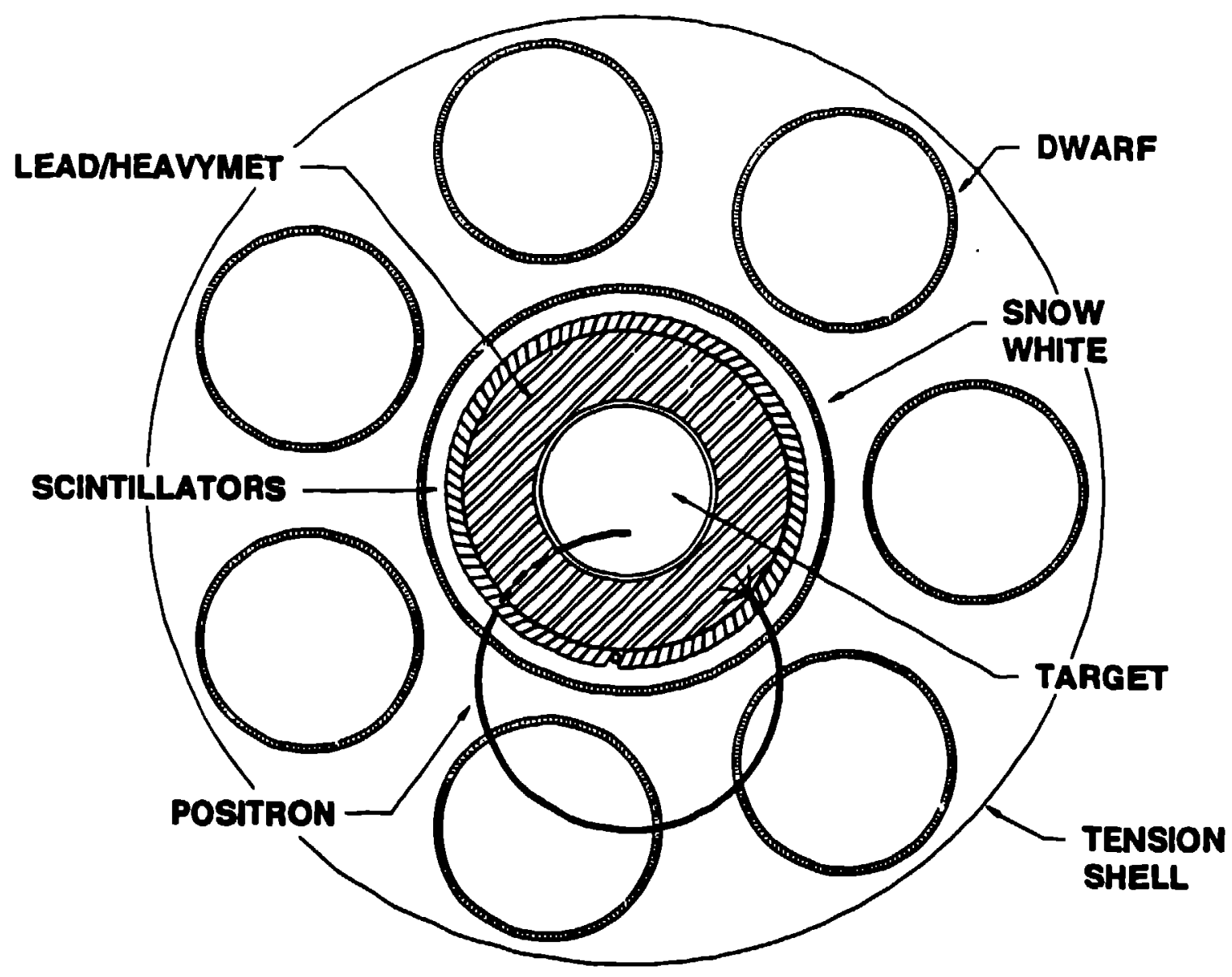

Figure 1 


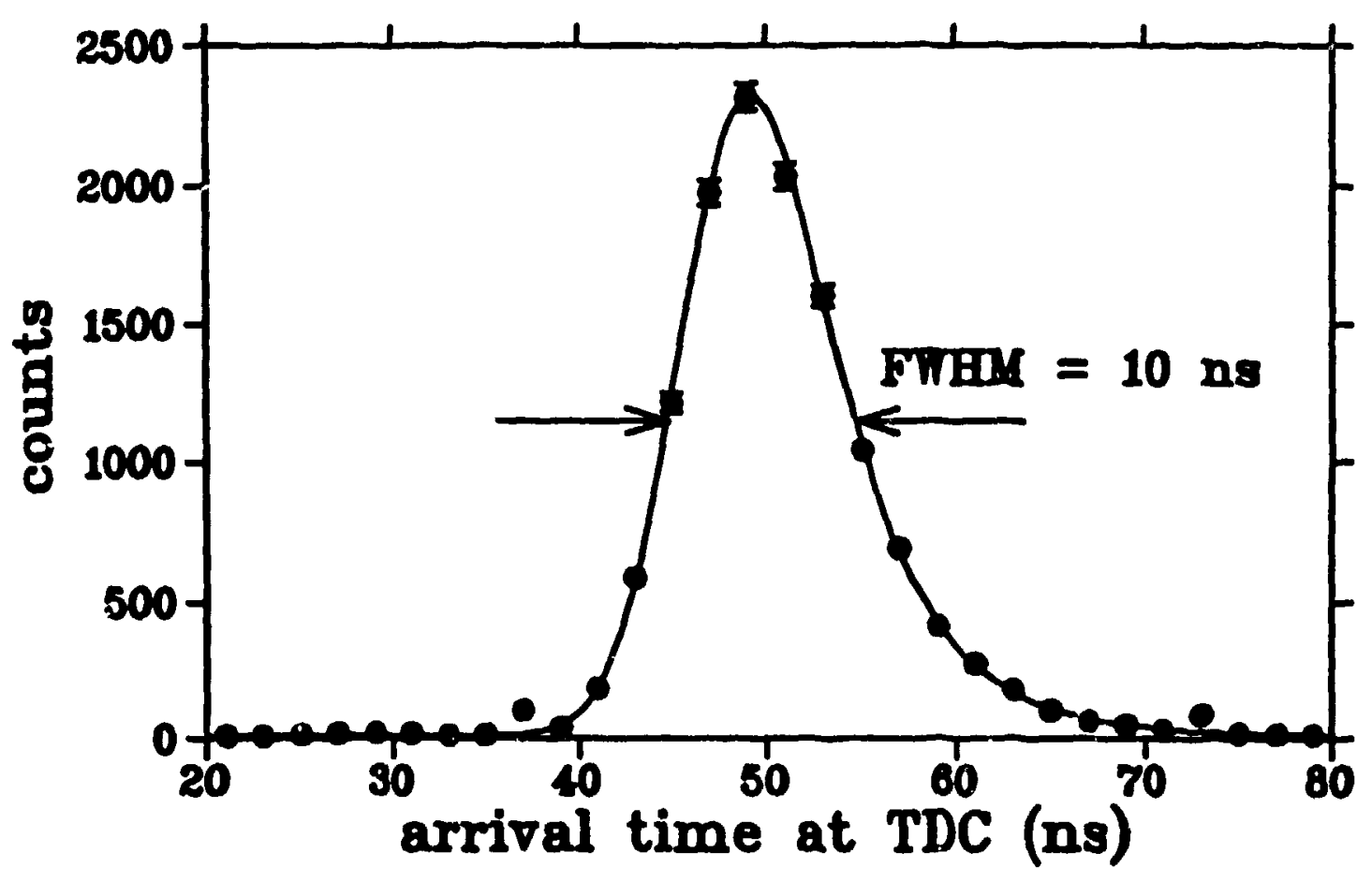

Lin.., 


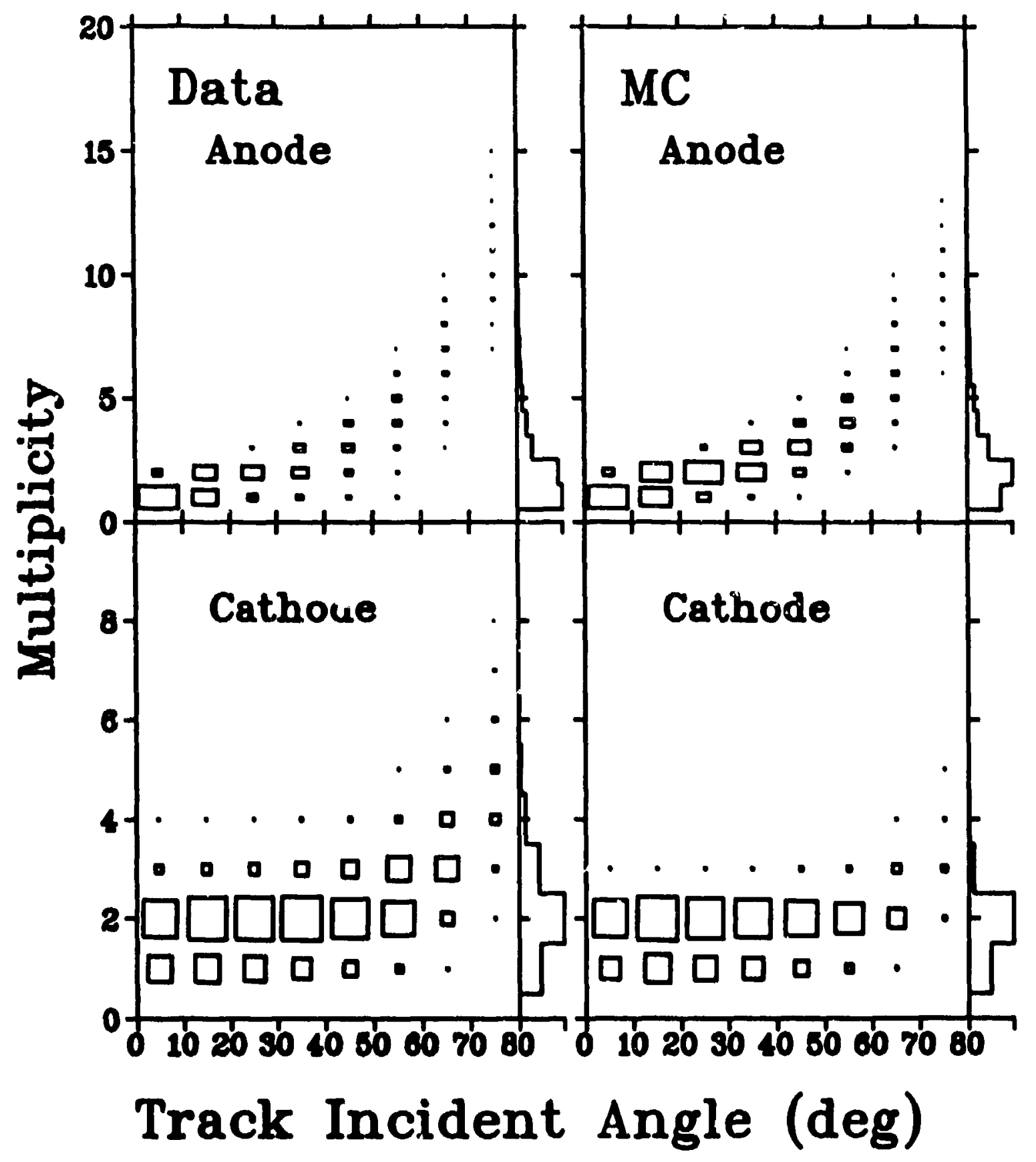

Figure 3 

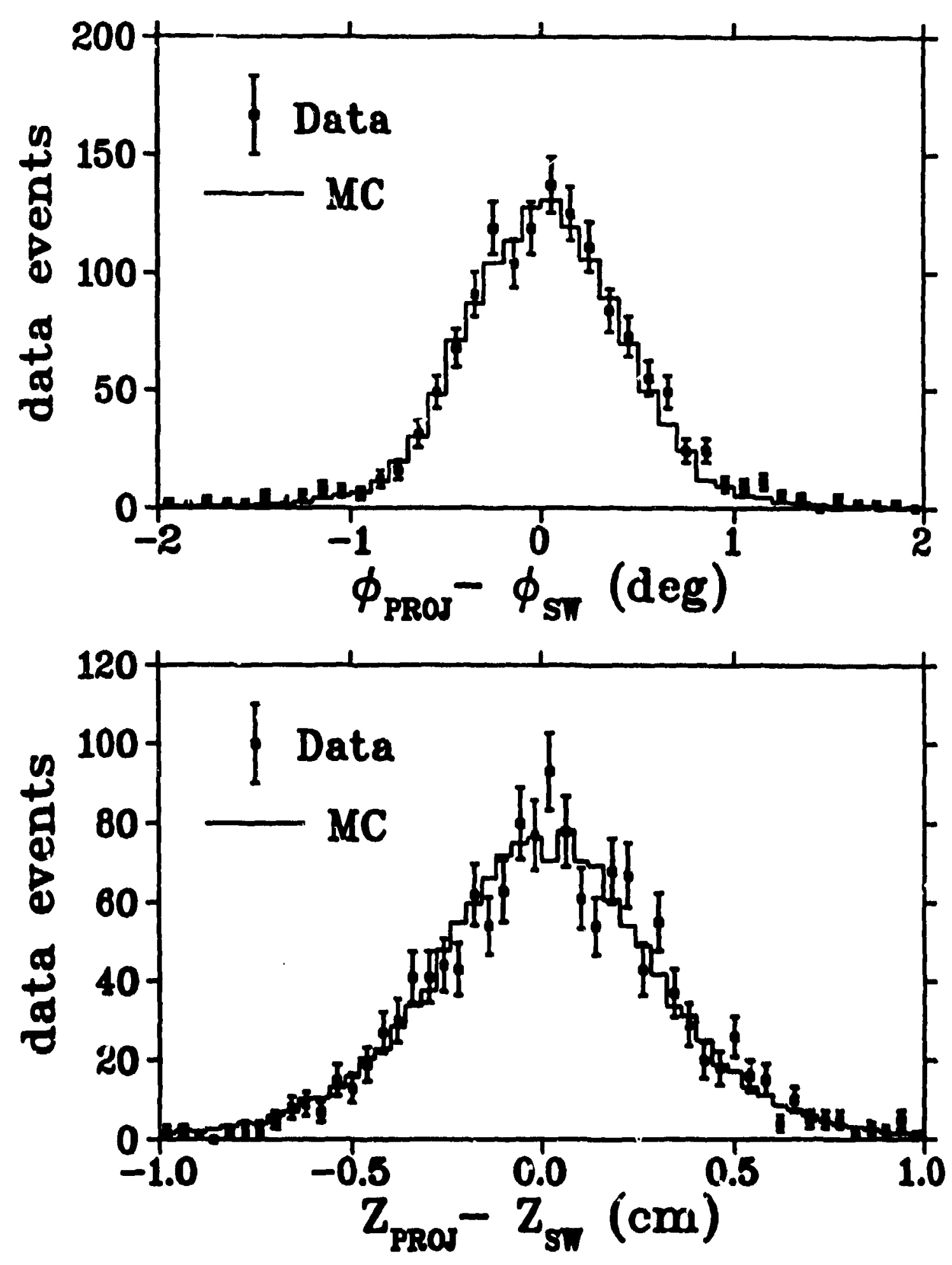


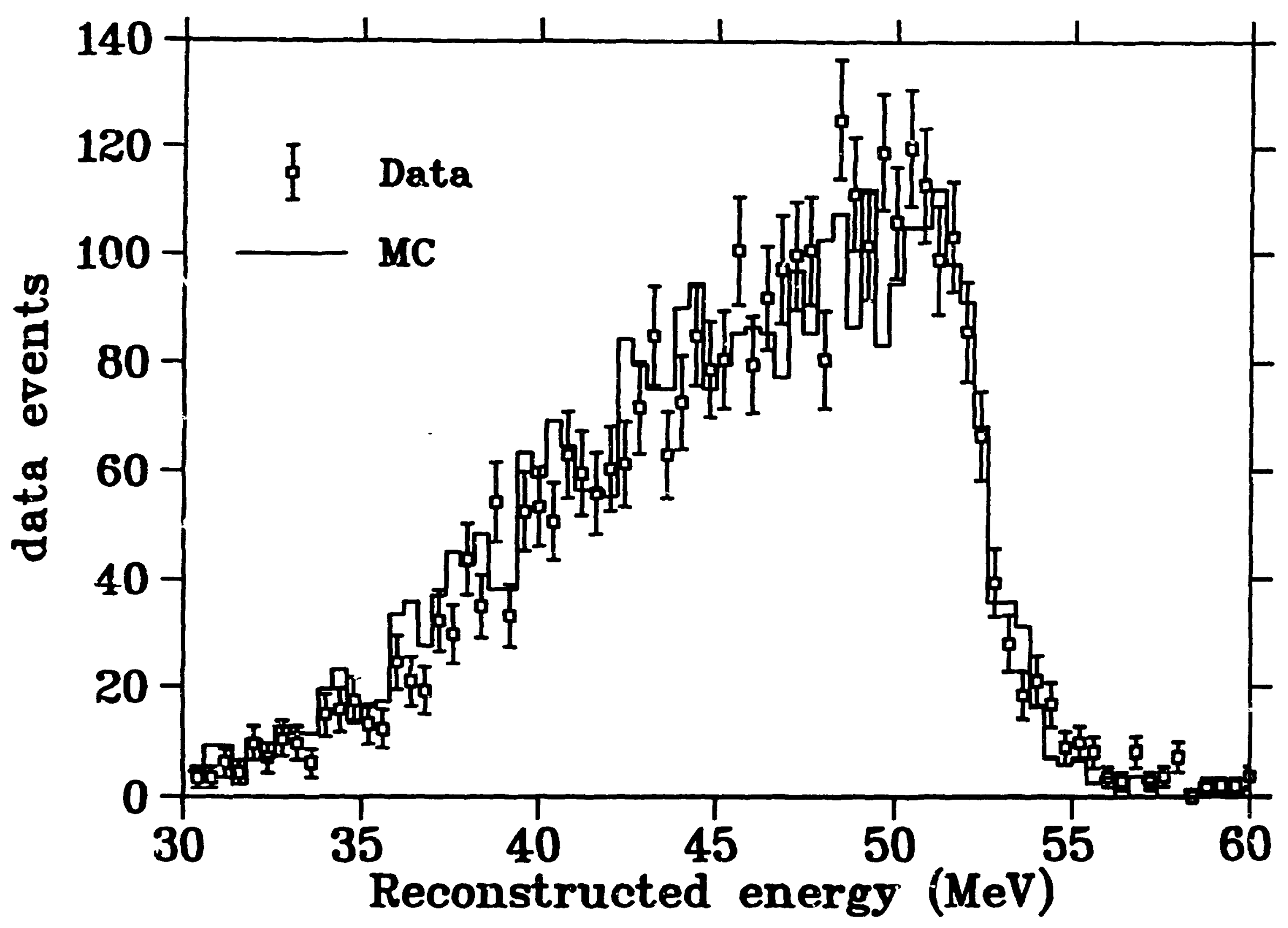




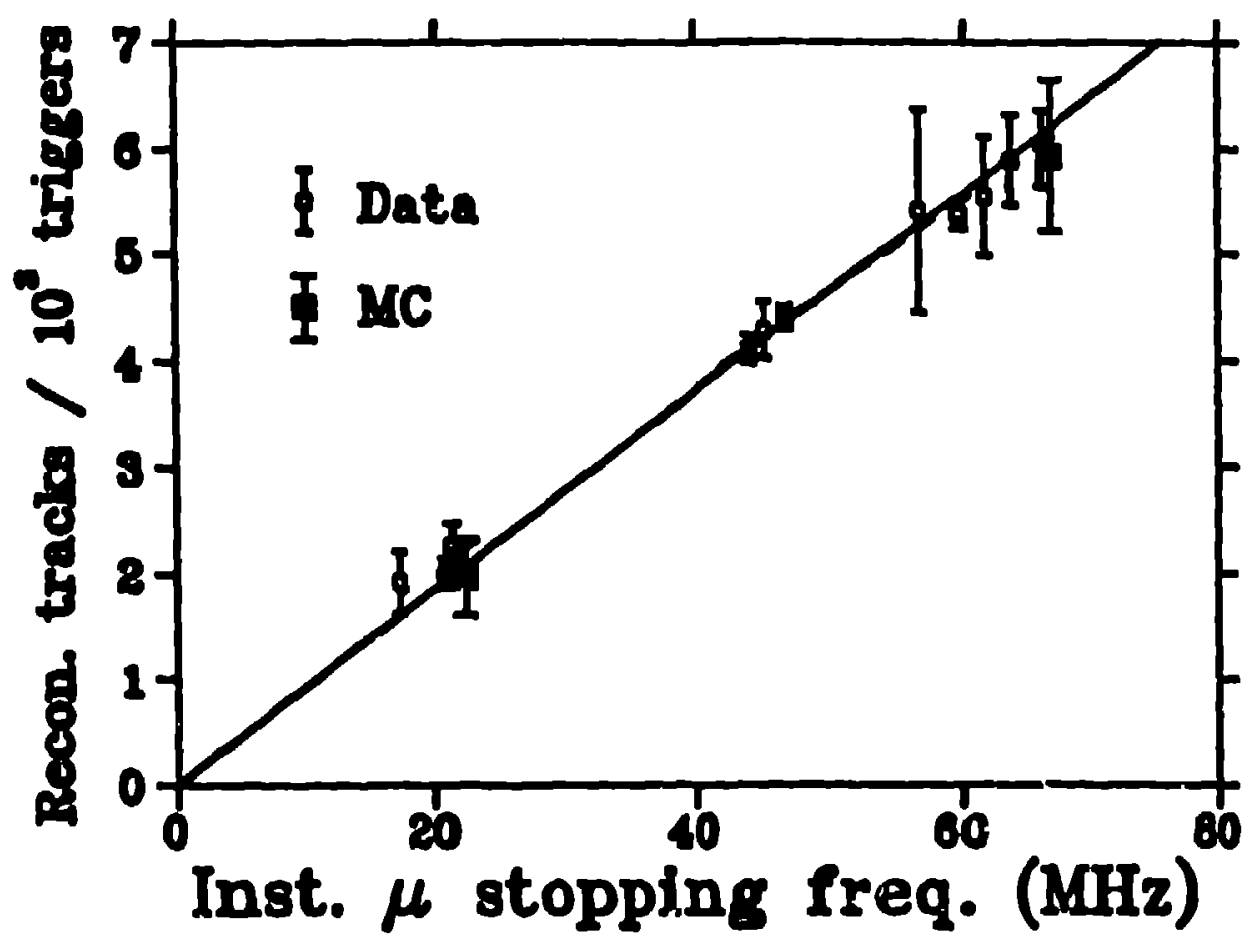

